\title{
Gestão de resíduos sólidos urbanos em municípios do Brasil: Uma revisão dos métodos de avaliação
}

\author{
Paula Thaise Bermudez dos REIS'; Ubirajara Aluízio de Oliveira MATTOS'; \\ EImo Rodrigues da SILVA \\ $\triangle$ paulatbdr@hotmail.com
}

1. Universidade do Estado do Rio de Janeiro, Rua São Francisco Xavier, 524, Rio de Janeiro, Brasil.

\section{Histórico do Artigo:}

Recebido: 14 de janeiro de 2016
Aceito: 23 de novembro de $2016 \quad$ Publicado: 22 de dezembro de 2016

\begin{abstract}
Resumo: Um dos maiores desafios da humanidade no século XXI é promover uma gestão adequada dos resíduos sólidos, a qual pressupõe a sua avaliação e monitoramento a fim de formular estratégias, promover a sustentabilidade ambiental e a melhoria da qualidade de vida da população. Este trabalho tem como objetivo realizar uma revisão sistemática da literatura referente às metodologias empregadas em estudos avaliativos sobre gestão de resíduos sólidos urbanos (RSU) em municípios brasileiros, caracterizando o perfil dessas publicações. Para tanto, foram realizadas buscas no Portal Periódicos Capes utilizando-se palavras-chave relacionadas ao tema. Após as triagens, foram selecionados 29 artigos, sendo que apenas cinco deles apresentaram métodos qualiquantitativos, passíveis de serem aplicados em outros municípios. Foi possível verificar a necessidade de aprimoramento e atualização dos itens de análise empregados nestas avaliações, visando adequá-los aos princípios e elementos da Política Nacional de Resíduos Sólidos.
\end{abstract}

Palavras-chave: Gerenciamento, Políticas públicas, Métodos de avaliativos.

\section{Waste management in cities of brazil: A review of assessment methods}

\begin{abstract}
One of the greatest challenges facing humanity in the twenty-first century is to promote a proper management of solid waste, that requires assessment and monitoring, to formulate strategies, promoting environmental sustainability and improving the population's quality of life. This study aims to systematically review the literature about methods used in evaluation studies on municipal solid waste management (MSW) in Brazilian cities and to characterize these publications. For this, searches were conducted in Portal Capes using keywords related to the topic. After the screening, 29 articles were selected and only five of them showed qualitative and quantitative methods, which can be applied in other municipalities. It was possible to verify the need to improve and update the analysis items employed in this evaluations, in order to adapt them to the principles and elements of the National Solid Waste Policy.
\end{abstract}

Keywords: Management, Public policy, Evaluation methods. 


\section{Gestión de residuos sólidos municipales en brasil: Una revisión de los métodos de evaluación}

Resumen: Una de las mayores dificultades de la humanidad en el siglo XXI es promover el manejo adecuado de los residuos sólidos, lo que requiere su evaluación y monitorio con la finalidad de formular estrategias para promover la sostenibilidad ambiental y mejora de la calidad de vida de las personas. Este trabajo tiene como objetivo hacer una revisión sistemática de la literatura sobre los métodos utilizados en los estudios de evaluación sobre la gestión de residuos sólidos urbanos (RSU) en las ciudades brasileñas, describiendo el perfil de estas publicaciones. Para eso, búsquedas se realizaron en Portal Capes utilizando palabras clave relacionadas con el tema. Veinte y nueve artículos fueron seleccionados, sólo cinco de ellos mostraron métodos cualitativos y cuantitativos que sirven como modelos y pueden ser aplicadas en otros municipios. Fue posible verificar la necesidad de mejora y actualización de los elementos análisis de los empleados en estas evaluaciones, para ajustarlos a los principios y elementos de la Política Nacional de Residuos Sólidos.

Palabras clave: Gestión, Políticas públicas, Métodos de evaluación.

\section{INTRODUÇÃO}

Um dos maiores desafios da humanidade no século XXI é promover uma gestão adequada dos resíduos sólidos. № ano de 2013, 41,74\% de todo lixo coletado no Brasil ainda seguia para destinação final inadequada, ou seja, lixões ou aterros controlados (ABRELPE, 2014).

A gestão e a disposição inadequada dos resíduos sólidos causam impactos socioambientais, tais como: degradação do solo, poluição dos corpos hídricos, intensificação de enchentes, poluição atmosférica, proliferação de insetos vetores de doenças e catação em condições insalubres (BESEN et al., 2010).

A Política Nacional de Resíduos Sólidos (PNRS), Lei $n^{0}$. 12.305 (BRASIL, 2010), da Presidência da República, representa um marco legal da gestão e manejo dos resíduos sólidos. Ela fornece diretrizes para a gestão e o gerenciamento de resíduos, enfatizando a adoção de práticas hierárquicas durante esse processo, como: a não geração, a redução na fonte geradora, seguida pela reutilização, reciclagem e por último, o tratamento e a disposição final adequada dos resíduos.

A PNRS traz princípios e conceitos importantes para a gestão de resíduos, dentre outros, destacam-se a responsabilidade compartilhada e a logística reversa. A partir dessa lei, não somente o governo federal, estadual e municipal, mas também toda a cadeia de produção e consumo se torna responsável, de alguma forma, pela gestão do ciclo de vida dos produtos 
Gestão de resíduos sólidos urbanos em municípios do Brasil: Uma revisão dos métodos de avaliação

(R0MEIR0, 2011). Segundo Godoy (2013), embora esteja estabelecido este princípio da responsabilidade compartilhada, são as prefeituras as que possuem a maior participação e responsabilidade na gestão dos resíduos sólidos urbanos. Nos últimos anos, a ação municipal na área social e ambiental foi motivada pela descentralização de atribuições e competências das esferas federal e estadual para a municipal, justificada pelos governos locais estarem mais próximos das demandas da população (SILVA, 2011).

Aos municípios cabe elaborar e implementar seus Planos Municipais de Gestão Integrada de Resíduos Sólidos (PMGIRS), sendo esta a condição para terem acesso a recursos, incentivos ou financiamentos destinados à limpeza urbana e o manejo de resíduos sólidos.

A gestão de resíduos sólidos deve ser constantemente avaliada (SANTIAG0 e DIAS, 2012). A avaliação e monitoramento das políticas públicas municipais possibilitam reformular estratégias, promover a sustentabilidade ambiental e melhoria da qualidade de vida da população (NUNES JR.; FERNANDES, 2012). Historicamente, na administração pública brasileira não há a preocupação de se avaliar programas públicos (COSTA e CASTANHAR, 2003), no entanto essa necessidade está impressa na legislação.

A PNRS estabelece em seu artigo 19, inciso VI que os planos municipais deverão conter indicadores de desempenho operacional e ambiental dos serviços públicos de limpeza urbana e de manejo de resíduos sólidos. Esses indicadores são elaborados com objetivo de monitorar e avaliar os resultados produzidos com a implementação dos programas e ações de gestão de resíduos desenvolvidos no município (MMA, 2011).

Veiga et al. (2014) analisaram indicadores propostos para a gestão de resíduos apresentados pelo Sistema Nacional de Informações sobre Saneamento (SNIS) e pela Associação Brasileira de Empresas de Limpeza Pública e Resíduos Especiais (ABELPRE), a partir desse estudo, constataram um avanço na elaboração de indicadores para gestão de resíduos sólidos urbanos e ressaltaram haver uma predominância na dimensão econômica. Segundo os autores, o desenvolvimento de novos estudos na área pode auxiliar na construção de indicadores para a gestão de resíduos segundo as diretrizes da Política Nacional de Resíduos Sólidos, visando o alcance de melhores condições ambientais e de saúde no contexto atual.

Destaca-se, também, como um dos princípios da PNRS, em seu Capítulo II, Art. $3^{\circ}$, item VI, o direito ao controle social o qual se define como: 
0 conjunto de mecanismos e procedimentos que garantam à sociedade informações e participação nos processos de formulação, implementação e avaliação das políticas públicas relacionadas aos resíduos sólidos (BRASIL, 2010).

Neste sentido, cabe destacar a importância dos estudos acadêmicos para monitorar e avaliar os impactos das políticas públicas, em particular, com relação à gestão dos resíduos urbanos. Contudo, tanto no Brasil, como na América Latina esses estudos são escassos, isto se dá, em parte, por existirem poucos centros de pesquisas que tratam a questão, pelo pouco interesse que os resíduos comuns despertam nos profissionais e pesquisadores, assim como pela pouca atenção dada pelo poder público às questões de saúde em geral e que repercute também no setor específico dos resíduos (Ferreira e Anjos, 2001; DIAZ et al., 1997).

Este trabalho tem como objetivo realizar uma revisão sistemática da literatura referente às metodologias empregadas em estudos avaliativos sobre a gestão de resíduos sólidos urbanos (RSU) em municípios brasileiros, caracterizando o perfil dessas publicações.

\section{MATERIAL E MÉTODOS}

Foram realizadas pesquisas no Portal Periódicos Capes, que oferece acesso a textos completos disponíveis em mais de 37 mil publicações periódicas, internacionais e nacionais, bem como diversas bases de dados que reúnem desde referências e resumos de trabalhos acadêmicos e científicos até normas técnicas, patentes, teses e dissertações dentre outros tipos de materiais, cobrindo todas as áreas do conhecimento (CAPES, 2014). Como palavras-chave foram utilizados os termos: Gestão municipal de resíduos sólidos, Gestão de resíduos, Gerenciamento de resíduos, Avaliação da gestão de resíduos e Municipal waste management Brazil. A busca realizada com tais palavras-chave ocorreu em: títulos, resumos (abstracts), palavras-chave (keywords). Não houve delimitação do período de pesquisa, de forma a obter o maior número de trabalhos. Foram encontradas 1971 publicações, após uma primeira triagem realizada através da leitura dos títulos, foram obtidos 60 trabalhos. Realizou-se então a leitura do título e do resumo de cada um dos 60 trabalhos, após isso foram selecionados 29 que mais se assemelhavam ao tema que se propunha estudar. Esses trabalhos foram analisados em profundidade, tendo como critérios de análise: o objetivo da pesquisa e a metodologia utilizada, sendo dado um enfoque especial aos artigos que desenvolveram novos modelos para a avaliação da gestão municipal de resíduos sólidos. 
Gestão de resíduos sólidos urbanos em municípios do Brasil: Uma revisão dos métodos de avaliação

\section{RESULTADOS E DISCUSSÃ0}

0s 29 trabalhos analisados foram publicados de 2003 a 2013, eles abordavam a gestão RSU em municípios de 10 estados brasileiros: Amazonas (1), Bahia (2), Minas Gerais (2), Pará (1), Paraíba (1), Paraná (4), Rio Grande do Norte (1), Rio Grande do Sul (2), Tocantins (1) e São Paulo (14), com maior percentual de publicações (figura 1).

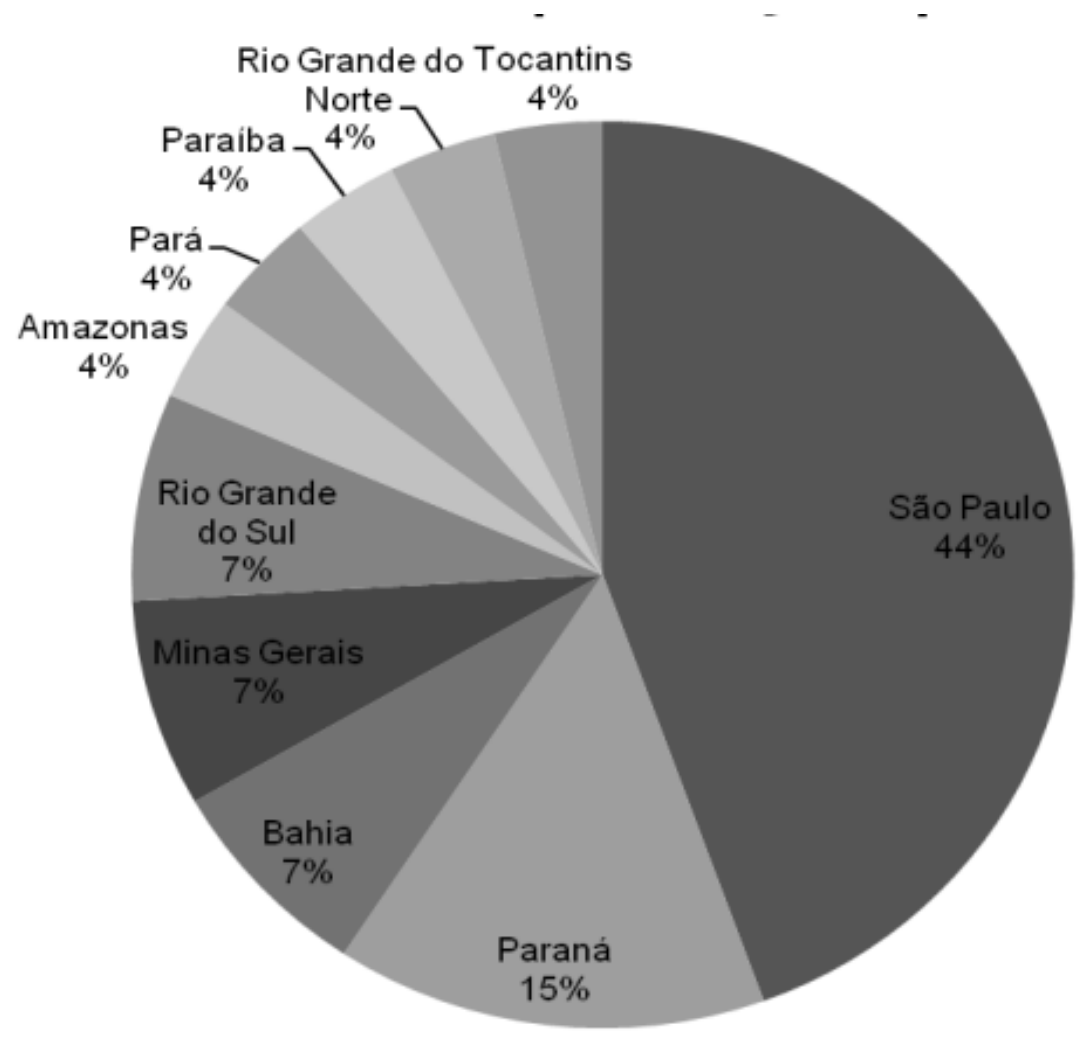

Figura 1. Estados alvo de estudos avaliativos sobre a gestão municipal de resíduos sólidos urbanos

Fonte: 0 s autores.

Quanto às instituições de pesquisa participantes das publicações podem ser listadas: Escola Agrotécnica Federal de Araguatins (EAFA), Escola Superior de Sustentabilidade (ESS), Faculdade Ages (AGES), Faculdade Estadual de Educação Ciências e Letras de Paranavaí (FAFIPA), Instituto Federal do Paraná (IFPR), Universidade de Brasília (UnB), Universidade de São Paulo (USP), Universidade Estadual da Paraíba (UEBP), Universidade Estadual de Campinas (Unicamp), Universidade Estadual de Feira de Santana (UEFS), Universidade Estadual de Maringá (UEM), Universidade Estadual Paulista (Unesp), Universidade Federal de Campina Grande (UFGC), 
Universidade Federal de Mato Grosso do Sul (UFMS), Universidade Federal de Minas Gerais (UFMG), Universidade Federal de São Carlos (UFSCar), Universidade Federal de Uberlândia (UFU), Universidade Federal do Maranhão (UFMA), Universidade Federal do Pará (UFPA), Universidade Federal do Rio Grande do Norte (UFRN), Universidade Feevale (FEEVALE) e Universidade Positivo (UP). Destas, a USP, UNESP, UNICAMP e UEM foram as que participaram com um maior número de trabalhos. As três instituições paulistas: USP, Unicamp e Unesp lideram o ranking das universidades brasileiras que mais publicaram artigos científicos entre os anos de 2010 e 2014 (SCIMAG0 Lab, 2015). Não foram obtidas produções de instituições de Ensino Superior de várias unidades federativas do país, tais como: Rio de Janeiro, Santa Catarina entre outros.

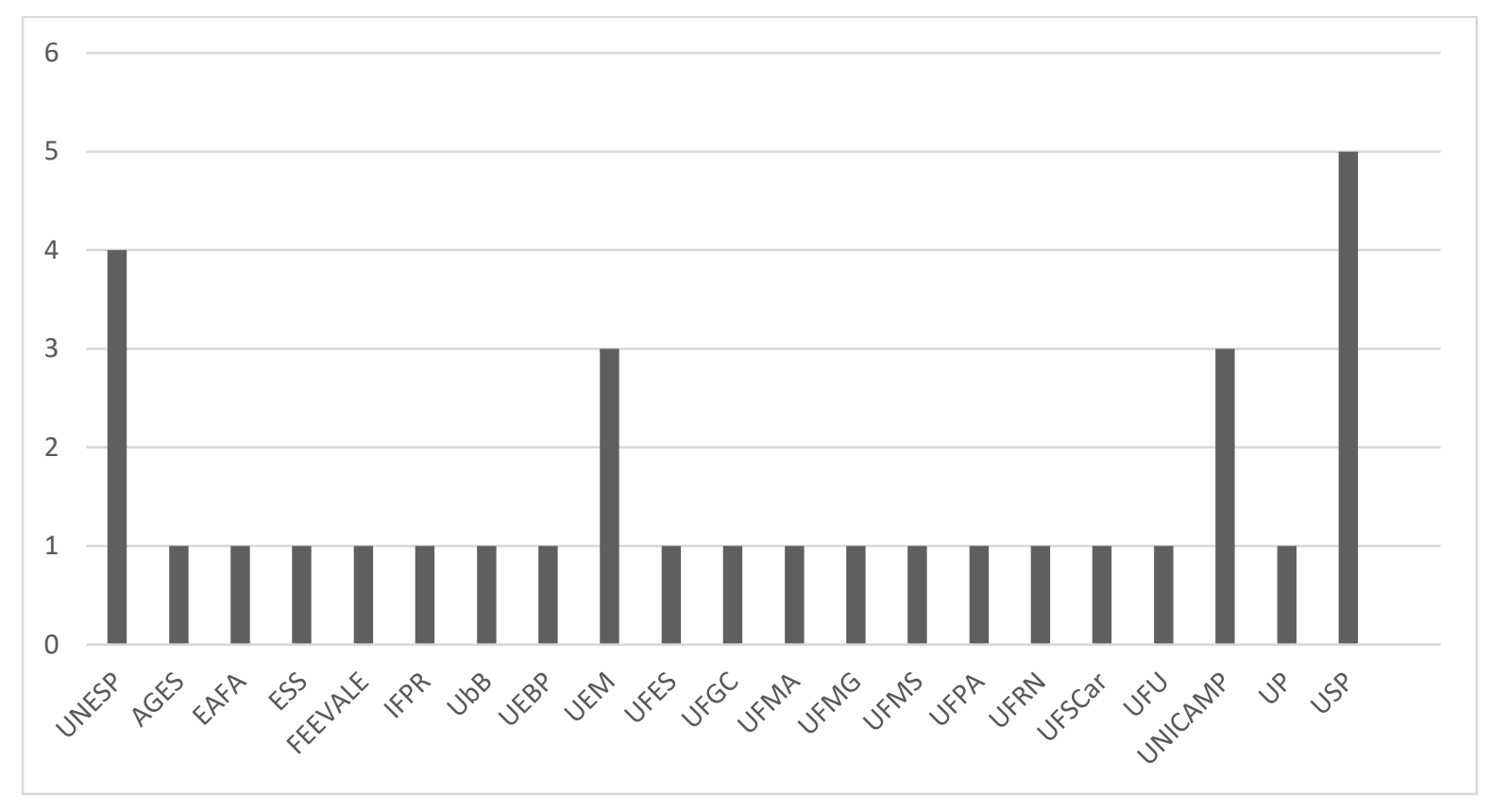

Figura 2. Número de publicações em que cada Instituição participou

Fonte: 0s autores..

Dentre os 29 trabalhos analisados, 23 deles utilizaram como procedimentos metodológicos, revisão bibliográfica, observação direta, entrevistas e análises documentais, e analisaram apenas qualitativamente os cenários encontrados nos municípios pesquisados. Cinco trabalhos realizaram estudos quali-quantitativos, sendo eles abordados a seguir.

Ferraz (2008) elaborou um índice de gestão de resíduos (IGR), fundamentado nos conceitos de gestão integrada de resíduos sólidos, sustentabilidade, indicadores e requisitos para sistema de gestão, encontrados na literatura. 0 modelo é estruturado em quatro dimensões: Estratégica, Coleta e Transporte, Triagem e Tratamento e Destinação Final. Para 
Gestão de resíduos sólidos urbanos em municípios do Brasil: Uma revisão dos métodos de avaliação

cada dimensão foram definidos fatores de avaliação e para cada um dos fatores, itens de análise, totalizando 192. 0 índice varia de 0 a 1 e é determinado através da comparação entre 0 percentual de pontuação do município ideal e o percentual de pontuação obtido pelo município estudado. Um questionário foi utilizado para o preenchimento da matriz de avaliação. Por tratar-se de um modelo relativamente simples, permitiu que o município realizasse uma autoavaliação, identificando os aspectos positivos e negativos no gerenciamento dos resíduos. Embora tenha sido elaborado antes da PNRS, envolve em seus tópicos: a presença de indicadores de desempenho por parte do município, a inclusão dos catadores, participação da sociedade e logística reversa.

Polaz e Teixeira (2009) propuseram indicadores de sustentabilidade para a gestão de RSU em São Carlos (SP). Segundo os autores, sua utilização constitui uma forma de operacionalizar o conceito de sustentabilidade, sendo esses indicadores instrumentos de mensuração, capazes de prover informações, que facilitem a avaliação do grau de sustentabilidade das sociedades, o monitoramento das tendências de seu desenvolvimento e a definição de metas de melhoria. 0 modelo foi elaborado sob a perspectiva de cinco dimensões: ambiental, econômica, social, política e cultural. Os indicadores foram desenvolvidos a partir da identificação dos problemas prioritários para a gestão de RSU por meio de consulta a gestores municipais, que resultou em um conjunto de 15 indicadores, cujos valores expressam sua tendência favorável, desfavorável ou muito desfavorável à sustentabilidade. Pinho (2011) observou que estes indicadores não contemplam a legislação e outros instrumentos no plano nacional assim como a interação entre as administrações nos três níveis de governo.

Hamada (2011) formulou um Índice de Qualidade para RSU, baseado em um modelo multicriterial, fundamentado na estratégia geral de gestão, dispondo-se em dois níveis hierárquicos, o Grupo Prioritário (Recurso Humano, Legislação e Planejamento e Ações) e Específico (delimitações dentro do Prioritário). Para cada grupo prioritário foram selecionados três indicadores, ou ações com graus de importância definidos através do método Analytic Hierarchy Process (AHP), no qual foram consultados 14 especialistas na área de gestão de RSU. 0 índice varia de 0 a 1. Este modelo é semelhante ao de Ferraz (2008), no entanto, utiliza uma matriz de avaliação pouco detalhada, contendo apenas nove indicadores. Confrontando-a com a PNRS, observa-se basicamente a presença de plano de RSU, licença para o aterro sanitário, coleta seletiva e triagem dos resíduos, não sendo abordados, por exemplo, aspectos importantes como a logística reversa ou programas educativos. 
Pinho (2011) propôs um método de avaliação de Planos de Gestão Integrada de RSU que contempla dois momentos: a sua concepção, tendo como parâmetro o art. 19 da PNRS, averiguando o percentual de conformidades; e implantação, quando desenvolveu um modelo estruturado em seis módulos com o mesmo peso: Recursos humanos, Potencialidade gerencial, Satisfação do cliente, Benefícios sociais, Econômico-financeiro e Operacional. Consiste em uma matriz simples, composta por 18 itens. Para cada item há uma pergunta, para a qual deverá ser respondido sim ou não. Completado seu preenchimento é possível averiguar o percentual de conformidades. As informações sobre cada município analisado foram obtidas através de revisão bibliográfica, visitas de campo e entrevistas aos secretários responsáveis pela gestão de RSU. 0 modelo é simples, sendo desta forma de fácil aplicabilidade.

Santiago e Dias (2012) elaboraram uma matriz de indicadores de sustentabilidade, construída a partir de informações obtidas em referências nacionais e internacionais. Os indicadores foram organizados em uma matriz preliminar, a qual passou por um processo de validação externa, com especialistas, por meio do método Delphi. A matriz final possui seis dimensões de sustentabilidade: política, tecnológica, econômico-financeira, ambiental/ecológica, conhecimento e inclusão social, contemplando 42 indicadores e 126 descritores. Para cada dimensão foram elaboradas perguntas-chave. Para cada pergunta chave há indicadores, e para cada indicador, descritores. Cada descritor possui uma nota. Diferente de Polaz e Teixeira (2009), que realizaram um levantamento dos problemas prioritários no município estudado para elaboração de indicadores, Santiago e Dias (2012) utilizaram indicadores constantes na literatura para elaboração de sua matriz avaliativa, que incorpora elementos importantes da PNRS, como a inclusão social de catadores, educação ambiental e mobilização social. No entanto, também não há menção à logística reversa.

\section{CONCLUSÃ0}

A academia tem papel relevante para 0 controle social no que se refere à avaliação da gestão de resíduos sólidos urbanos em municípios do Brasil. No entanto, esta atividade não deve se limitar às instituições de pesquisa, e sim ser uma prática comum nos municípios, como o já previsto na legislação e que é fundamental para a eficiência na gestão. Há um número restrito de métodos avaliativos da gestão de RSU no Brasil, parte deles incompletos e/ou desatualizados, desta forma, novos estudos desenvolvendo matrizes de avaliação simples, atendendo aos requisitos da PNRS e abordando a gestão de forma integrada e holística fazem-se necessários. 
Gestão de resíduos sólidos urbanos em municípios do Brasil: Uma revisão dos métodos de avaliação

A ausência do registro de produções de instituições em vários estados brasileiros não significa a inexistência dos mesmos, isto pode ser atribuído ao fato de não estarem presentes na base de dados apreciada neste estudo ou mesmo em haver pouca divulgação desses trabalhos.

Observou-se a ausência da logística reversa, nos instrumentos avaliativos, sua implementação no país ainda é um desafio. Este item deve ser incluído em novos modelos, pois o poder púbico local tem papel fundamental neste processo.

\section{REFERENNCIAS}

Associação Brasileira de Empresas de Limpeza Pública e Resíduos Especiais. ABRELPE. Panorama de Resíduos Sólidos no Brasil- 2013. São Paulo: Abrelpe, 2014.

ANDRADE, T. R.; SILVA, C. E. Analysis of sustainability in solid waste management in the city: the case of Paripiranga, Brazil. Revista Ibero-Americana de Ciências Ambientais, Aquidabã, v.3, n.2, p.91-111, 2012.

BRASIL, SENAD0 FEDERAL. Lei Nº 12.305, de 02 de agosto de 2010. -Institui a Política Nacional de Resíduos Sólidos. Brasília: Gráfica do Senado, 2010. p. 18.

Coordenação de Aperfeiçoamento de Pessoal de Nível Superior- CAPES. Disponível em: 〈http://www.capes.gov.br/> Acesso em Novembro, 2014.

COSTA, F. L. e CASTANHAR, J. C., Avaliação de programas públicos: desafios conceituais e metodológicos. Revista de Administração Pública, 5, 2003.

DIAZ, L. F.; SAVAGE, G. M.; EGGERTH, L. L., 1997. Managing solid wastes in developing countries. Waste Management, v. 10 , p. $43-45$.

FERRAZ, J.L. Modelo para avaliação da gestão municipal integrada de resíduos sólidos urbanos. 2008. Tese (Doutorado em Engenharia Mecânica) Universidade Estadual de Campinas, Campinas, 2008.

Ferreira, J. A. Anjos L. A. Aspectos de saúde coletiva e ocupacional associados à gestão dos resíduos sólidos municipais. Cadernos de Saúde Pública; v. 17.p.689-96, 2001.

Godoy, M. B. R. B. (2013) Dificuldades para aplicar a Lei da Política Nacional de Resíduos Sólidos no Brasil. Caderno de Geografia. V. 23, n. 39, p.1-12.

HAMADA, P. Formulação de um índice de qualidade de gestão de resíduos sólidos urbanos. 111 f. Dissertação (Mestrado em Engenharia) - Universidade Estadual Paulista Júlio de Mesquita Filho Bauru. 2012.

MINISTÉRIO DO MEIO AMBIENTE (Brasil). Guia para elaboração dos Planos de Gestão de Resíduos Sólidos, 2011.

NUNES, M. R.; JR., A. P.; FERNANDES, V. Gestão Ambiental Municipal : objetivos, instrumentos e agentes. Revista Brasileira de Ciências Ambientais, v. 23, p. 66-72, 2012. 2, 2012.

PINHO, P. M. Avaliação dos planos municipais de gestão integrada de resíduos sólidos urbanos na Amazônia Brasileira. Tese (Doutorado em Ciência Ambiental) - Universidade de São Paulo. São Paulo. 2011.

POLAZ, C. N. Marcolino; TEIXEIRA, B. A. N. Indicadores de sustentabilidade para a gestão municipal de resíduos sólidos urbanos: um estudo para São Carlos (SP). Eng. Sanit. Ambient., Rio de Janeiro, v. 14, n. 3, 2009. 
R0MEIR0, T. C. Impactos e oportunidades do novo Marco Legal para os Resíduos Sólidos. Pontes, São Pulo, v. 7 , n.2 2011.

SANTIAG0, L. S.; DIAS, S. M. F. Matriz de indicadores de sustentabilidade para a gestão de resíduos sólidos urbanos. Eng. Sanit. Ambient., Rio de Janeiro, v. 17, n.

Scimago LAB. Scimago Institutions Rankings. Disponível em: http://www.scimagoir.com/research.php. Acesso em: Março, 2015.

SILVA, F. E. C. E. Gerenciamento de Resíduos Sólidos de Jundiaí - SP. Revista Brasileira de Ciências Ambientais, v. 22 , p. 38-49, 2011.

VEIGA, et al. Análise de indicadores propostos para gestão de resíduos sólidos urbanos no Brasil. In: Convibra Administração, 2014. 